\title{
In vitro bond strength and fatigue stress test evaluation of different adhesive cements used for fixed space maintainer cementation
}

\author{
Kenan Cantekin ${ }^{1}$, Ebru Delikan $^{1}$, Secil Cetin $^{1}$
}

Correspondence: Dr. Kenan Cantekin Email: k_cantekin@hotmail.com
'Department of Pediatric Dentistry, Faculty of Dentistry, Erciyes University, Kayseri, Turkiye

\begin{abstract}
Objective: The purposes of this research were to (1) compare the shear-peel bond strength (SPBS) of a band of a fixed space maintainer (SM) cemented with five different adhesive cements; and (2) compare the survival time of bands of SM with each cement type after simulating mechanical fatigue stress. Materials and Methods: Seventy-five teeth were used to assess retentive strength and another 50 teeth were used to assess the fatigue survival time. SPBS was determined with a universal testing machine. Fatigue testing was conducted in a ball mill device. Results: The mean survival time of bands cemented with R \& D series Nova Glass-LC (6.2 h), Transbond Plus (6.7 h), and $\mathrm{R} \& \mathrm{D}$ series Nova Resin $(6.8 \mathrm{~h})$ was significantly longer than for bands cemented with Ketac-Cem $(5.4 \mathrm{~h})$ and GC Equia $(5.2 \mathrm{~h})(P<0.05)$. Conclusion: Although traditional glass ionomer cement $(\mathrm{GIC})$ cement presented higher retentive strength than resin-based cements (resin, resin modified GIC, and compomer cement), resin based cements, especially dual cure resin cement (nova resin cement) and compomer (Transbond Plus), can be expected to have lower failure rates for band cementation than GIC (Ketac-Cem) in the light of the results of the ball mill test.
\end{abstract}

Key words: Shear bond strength, space maintainers, transbond

\section{INTRODUCTION}

Space management is an important responsibility of clinicians who are involved in monitoring developing dentition, as the loss of arch length may lead to problems such as crowding, ectopic eruption, dental impaction, crossbite formation, and dental centerline discrepancies. ${ }^{[1]}$

The use of space maintainers (SMs) may potentially obviate the need for later extractions and/or complex orthodontic treatment. ${ }^{[2]}$ SMs are broadly classified as fixed or removable appliances used to preserve arch length following premature loss or elective extraction of tooth/teeth. SM appliances are most commonly used to maintain the space created by early loss of a first or second primary molar while awaiting the eruption of its successor. ${ }^{[1,3,4]}$
The band and loop (B and L) SM is the most common type of SM used in the case of premature extraction of a primary molar ${ }^{[2,3]}$ because it can be produced easily and economically, it requires little chair time for application, it can be used bilaterally, and it's well-tolerated by children. Despite its unique combination of favorable properties, the B and L $\mathrm{SM}$ have some disadvantages. Cement failures and breakage of the solder joint or band in the B and L SM are two of its primary drawbacks. ${ }^{[2,3,5]}$

In last two decades, glass ionomer cements (GIC) have been very popular for fixed SMs with band cementation because they can adhere to enamel and metal, they can release fluoride ions, they can be bonded in wet conditions, and they have an antimicrobial effect. The main shortcoming of GICs is their susceptibility to moisture contamination during the setting time,

\footnotetext{
How to cite this article: Cantekin K, Delikan E, Cetin S. In vitro bond strength and fatigue stress test evaluation of different adhesive cements used for fixed space maintainer cementation. Eur J Dent 2014;8:314-9.

Copyright @ 2014 Dental Investigations Society. DOI: $10.4103 / 1305-7456.137632$
} 
and the maximum bond strength is obtained only after 24 h. $^{[6,7]}$

So far, comparative evaluation of retentive strength or survival times of different types of adhesive cements for SM cementation has not been documented.

The purposes of this research were to (1) compare the shear-peel bond strength (SPBS) of a band of a fixed SM cemented with five different adhesive cements: GICs (Ketac-Cem [3M ESPE, St Paul, Minn.] and GC Equia [GC, Tokyo, Japan]), resin modified GIC (RMGIC) (R \& D series Nova Glass-LC [Imicryl, Konya, Turkey]), compomer cement (Transbond Plus [3M Unitek, California, USA]) and dual cure resin cement ( $\mathrm{R} \& \mathrm{D}$ series Nova Resin [Imicryl, Konya, Turkey]); (2) assess the site of bond failure for each adhesive material and compare the amount of cement remaining on the teeth after debanding; and (3) compare the survival time of bands of SM with each cement type after simulating mechanical fatigue stress in a ball mill testing device.

\section{MATERIALS AND METHODS}

This research was approved by the ethical committee of the Faculty of Medicine of Erciyes University, Kayseri. A total of 125 extracted human mandibular third molars were used in this study. Seventy-five teeth were used to assess retentive strength and another 50 teeth were used to assess the fatigue survival time. The extracted teeth were stored in distilled water continuously after extraction. Teeth with hypoplastic enamel, cracks, caries, or restoration were not included in the study. Each of the 75 teeth was mounted vertically in a self-cure acrylic resin so that the crown was exposed. The sample was randomly divided into five groups of 15 each. The teeth were cleaned and polished with a slurry of nonfluoridated flour of pumice (Moyco Industries, Philadelphia, PA) for $10 \mathrm{~s}$ using a rubber prophylactic cup, and then were rinsed for $10 \mathrm{~s}$ with a stream of water and then dried. The acrylic blocks were number coded, with a different number for each group of samples.

The adhesive cements used in this study included conventional GICs (Ketac-Cem [3M ESPE, St Paul, Minn.], GC Equia [GC, Tokyo, Japan]), RMGIC (R \& D series Nova Glass-LC, Imicryl, Konya, Turkey), compomer cement (Transbond Plus, 3M Unitek, California, USA), and dual cure resin cement ( $\mathrm{R} \& \mathrm{D}$ series Nova Resin, Imicryl, Konya, Turkey).
A $0.9 \mathrm{~mm}$ (0.036 inch) stainless steel wire was attached to the mid-buccal and mid-lingual side with hard solder to load the cell with the universal unit. The modified bands of the SM were adapted to each tooth and the bands were cemented with adhesive cements according to manufacturer's instructions. The R \& D series Nova Glass-LC, transbond plus, and R \& D series Nova Resin were cured with a light emitting diode light cure (VALO LED, Ultradent, South Jordan, USA) with an intensity of $1200 \mathrm{mV} / \mathrm{cm}^{2}$ for $20 \mathrm{~s}$ from the occlusal surface. The Ketac-Cem and GC Equia were allowed to set for $10 \mathrm{~min}$. The specimens were then transferred to store at $37^{\circ} \mathrm{C}$ in $100 \%$ humidity for $24 \mathrm{~h}$, and they were subsequently tested for retentive strength.

\section{Shear-peel bond strength test}

For the SPBS test, each specimen was secured in a universal testing machine (Instron, AGS-1000 kg/W; Shimadzu Corp., Chiroda-Ku, Tokyo, Japan) and each specimen was loaded into the testing machine by means of a $0.9-\mathrm{mm}$ rigid stainless steel loop between the buccal and lingual surfaces [Figure 1]. Testing proceeded until the band was removed completely from the tooth. The maximum force required to remove the band was measured in Newtons $(\mathrm{N})$ $\left(1 \mathrm{MPa}=1 \mathrm{~N} / \mathrm{mm}^{2}\right)$, and the SPBS was then calculated by dividing the peak load values by the band surface area.

\section{Adhesive remnant index scores}

After the debanding procedure, samples were visually assessed at site of cement by one investigator and were classified with a modification of the adhesive remnant index (ARI) scores established by Artun and Bergland. ${ }^{[8]}$ The scoring was as follows: 0, no

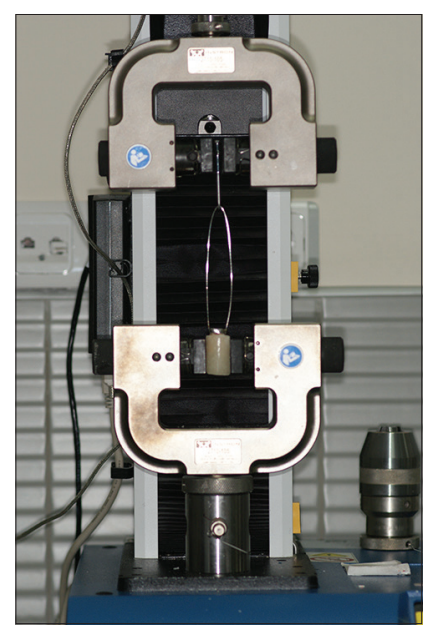

Figure 1: A specimen set-up in the Instron testing machine for a shearpeel bond strength test 
cement remains on the tooth surface; (1) less than half the crown surface under the band is covered by cement; (2) more than half the crown surface under the band is covered by cement; (3) the entire crown surface under the band is covered by cement.

\section{Evaluation of survival time}

To test survival time of the bands for different adhesive cements, another 50 extracted human mandibular third molars were collected and the teeth were cleaned and stored in a manner identical to that used for SPBS testing. The root surface of each tooth was coded with a diamond burr in the mid third to allow later identification. Bands were cemented with adhesive cements following the same cementation procedure as described previously. The samples were then transferred to a humidor set at $37^{\circ} \mathrm{C}$ for $24 \mathrm{~h}$. For each group, the samples were placed in a ball mill. This contained ceramic spheres and distilled water at $37^{\circ} \mathrm{C}$, rotating at 100 revolutions/min. After each hour of testing, samples were inspected, failed samples (those with loose bands) were removed from the mill, and testing was resumed until all samples had failed. After each inspection period $(1 \mathrm{~h})$, fresh distilled water at $37^{\circ} \mathrm{C}$ was placed in the ball mill.

\section{Statistical analysis}

All calculations were processed using the Statistical Package for the Social Sciences (SPSS) statistical software, version 16 (SPSS Inc., Chicago, IL, USA). The Kolmogorov-Smirnov test was used to test the normality of the distribution of the data. The means, minimums, maximums, and standard deviations were calculated. The mean bond strengths of the groups were compared using one-way analysis of variance (ANOVA) and Tukey's post-hoc test (honestly significant difference) was used for a two-by-two comparison (significance level, $P<0.05)$. For comparison of ARI scores, a Chi-square with Fisher exact test was used. A log rank and Kaplan-Meier estimates test were used to compare survival time distributions for band samples in the ball mill test.

\section{RESULTS}

The descriptive and comparative statistics of SPBS for each of the adhesive cements are given in Table 1. The ANOVA that compared the experimental groups revealed the presence of significant differences among the groups $(P<0.001)$. The highest bond strength value was recorded for the Ketac-Cem (11.1 MPa), followed by R \& D series Nova Resin (10.9 MPa),
R \& D series Nova Glass-LC (6.46 MPa), Transbond Plus (5.5 MPa), and GC Equia (5.1 MPa).

The mean retentive strength of bands cemented with Transbond Plus and GC Equia was significantly lower than that of bands cemented with either Ketac-Cem $(P=0.14)$ or $\mathrm{R} \& \mathrm{D}$ series Nova $\operatorname{Resin}(P=0.19)$. However, there was no significant difference in mean retentive strength values for bands cemented with Ketac-Cem, R \& D series Nova Resin, or R \& D series Nova Glass-LC $(P>0.05)$.

The ARI scores for all adhesive groups are presented in Table 2. The most common type of failure for all cement groups was at the tooth-cement interface, at $70 \%$ [ARI scores of 0 or 1; Table 2]. The Chi-square test demonstrated a statistically significant difference among the adhesive groups $(P<0.001)$. The groups cemented with Ketac-Cem, Transbond Plus, R \& D series Nova Resin, and GC Equia showed a higher prevalence of ARI score " 1 ", whereas the group cemented using R \& D series Nova Glass-LC showed a higher frequency of ARI score " 2 ".

\begin{tabular}{|c|c|c|c|c|c|c|}
\hline Cement & $N$ & Mean & SD & Minimum & Maximum & $\begin{array}{c}\text { Tukey } \\
\text { grouping }\end{array}$ \\
\hline Ketac-Cem & 15 & 11.1 & 3.9 & 5.2 & 19.7 & $B$ \\
\hline GC Equia & 15 & 5.1 & 1.2 & 2.7 & 7.4 & A \\
\hline $\begin{array}{l}R \& D \text { series } \\
\text { Nova Glass } L C\end{array}$ & 15 & 6.4 & 3.4 & 2.1 & 11.5 & $A, B$ \\
\hline $\begin{array}{l}\text { Transbond } \\
\text { Plus }\end{array}$ & 15 & 5.5 & 5.0 & 1.1 & 17.1 & $A$ \\
\hline $\begin{array}{l}R \text { \& D series } \\
\text { Nova Resin }\end{array}$ & 15 & 10.9 & 5.5 & 3.1 & 23.2 & B \\
\hline Total & 75 & 7.8 & 4.7 & 1.0 & 23.2 & \\
\hline
\end{tabular}

SD: Standard deviation, SM: Space maintainers

\begin{tabular}{|c|c|c|c|c|c|c|}
\hline $\begin{array}{l}\text { ARI } \\
\text { score }\end{array}$ & $\begin{array}{c}\text { Ketac- } \\
\text { Cem } \\
(\%)\end{array}$ & $\begin{array}{c}\text { GC } \\
\text { Equia } \\
(\%)\end{array}$ & $\begin{array}{c}\text { R \& D } \\
\text { Series } \\
\text { Nova } \\
\text { Glass-LC } \\
(\%)\end{array}$ & $\begin{array}{c}\text { Transbond } \\
\text { Plus (\%) }\end{array}$ & $\begin{array}{c}\text { R \& D } \\
\text { Series } \\
\text { Nova } \\
\text { Resin } \\
(\%)\end{array}$ & $\begin{array}{c}\text { Total } \\
(\%)\end{array}$ \\
\hline $\begin{array}{l}0 \text { (no } \\
\text { cement } \\
\text { on tooth) }\end{array}$ & $0(0)$ & $0(0)$ & $2(13)$ & $6(40)$ & $2(13)$ & $10(13)$ \\
\hline 1 & $10(75)$ & $10(75)$ & $3(20)$ & $9(60)$ & $11(73)$ & $43(57)$ \\
\hline 2 & $5(25)$ & $5(25)$ & $8(53)$ & $0(0)$ & $2(13)$ & $20(27)$ \\
\hline $\begin{array}{l}3 \text { (no } \\
\text { cement } \\
\text { on band) }\end{array}$ & $0(0)$ & $0(0)$ & $2(13)$ & $0(0)$ & $0(0)$ & $2(3)$ \\
\hline
\end{tabular}


The survival time distribution for bands cemented with adhesive cements during the ball mill test is shown in Figure 2. The test results demonstrated that the mean survival time of bands cemented with R \& D series Nova Glass-LC (6.2 h), transbond plus (6.7 h), and R \& D series Nova Resin (6.8 h) was significantly longer than for bands cemented with Ketac-Cem (5.4 h) and GC Equia (5.2 h) $(P<0.05)$. On the other hand, there was no significant difference in the mean survival times among transbond plus, $R$ \& D series Nova Glass-LC, and R \& D series Nova resin $(P$ $>0.05)$. Equally, the difference in mean survival times between bands cemented with Ketac-Cem and GC Equia did not reach a statistically significant level $(P$ $>0.05$ ).

\section{DISCUSSION}

Although many authors have discussed the indications, contraindications, considerations, and developments for the use of different new forms of fixed SMs such as fiber SMs, the B and L SM is still the most commonly used in the case of unilateral primary loose tooth. In addition, GIC cement is most widely accepted for the cementation of SM bands ${ }^{[9-11]}$ because it can adhere to both enamel and metal. It also provides fluoride release and can facilitate fluoride uptake. Despite these advantages, the use of GICs has not eliminated the problem of cement failure.

According to results of previous clinical research, long-term failure rates of B and L SMs have been reported as ranging from $29 \%$ to $57 \%$ at periods

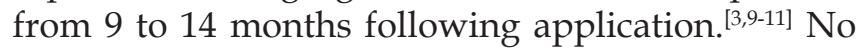
information exists, however, about the different adhesive systems for cementation, even though

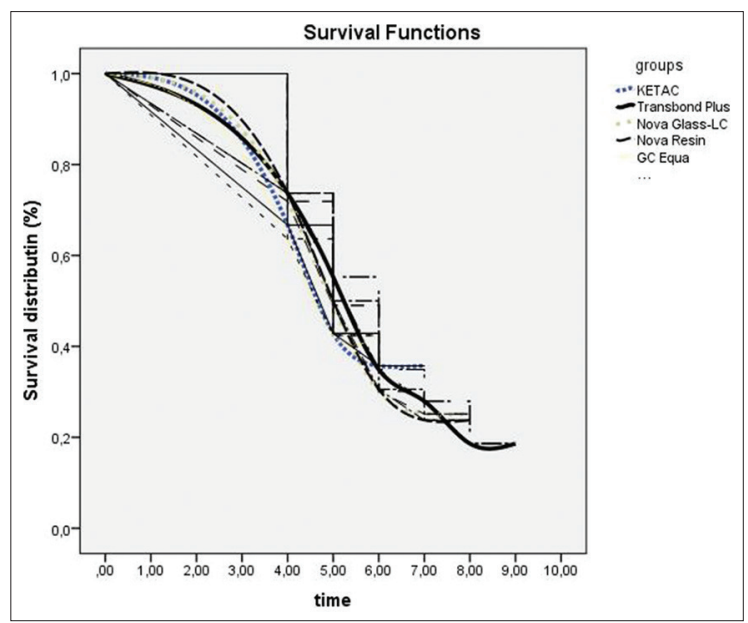

Figure 2: Survival time distribution of the space maintainer bands in each of the five adhesive groups the most frequent reason for failure of fixed SMs is a loss of cement around the SM band. ${ }^{[10,11]}$ Hence, the purpose of the present laboratory study was to compare retentive strength and survival time of SM bands cemented with GIC cements (Ketac-Cem and GC Equia), RMGIC cement (R \& D series Nova Glass-LC), compomer cement (transbond plus) and dual curable resin cement ( $R$ \& D series Nova Resin).

In this study, the mean retentive strength of SM bands cemented with transbond plus and GC Equia was significantly lower than that of bands cemented with either Ketac-Cem or R \& D series Nova Resin. Although R \& D series Nova Glass-LC showed lower strength value than Ketac-Cem and R \& D series Nova Resin, the differences did not reach a significant level $(P>0.05)$. No other study has compared these cements for band cementation, although similar products have been assessed. ${ }^{[12]}$ Comparisons can therefore be made only in the broadest sense. In accordance with the findings of the study reported here, Aggarwal et al. ${ }^{[12]}$ also found a significantly lower mean retentive strength for transbond plus cement compared with two RMGIC cements. Similarly, Gillgrass et al. evaluated band failure with different band cement materials and showed that the band failure rate of $2.8 \%$ for the conventional GIC (Ketac Cem) was lower than the $5 \%$ band failure rate for the compomer cement. ${ }^{[13]}$

For samples tested in present study, bond failure occurred predominantly at the enamel-cement interface. The amount of cement remaining on the enamel after debanding differed significantly for bands cemented with R \& D series Nova Glass-LC and all other adhesive cements. Although most bands cemented with Ketac-cem, transbond plus, R \& D series Nova Resin, and GC Equia had a cement remnant score of 0 and 1 , indicating that less than half of the crown surface under the band was covered by cement, most of the bands cemented with $\mathrm{R} \& \mathrm{D}$ series Nova Glass-LC had a cement remnant score of 2 or 3 , indicating that more than half the crown surface under the band was covered by cement. Previous studies that evaluated the debanding locations of RMGICs showed conflicting results. In those studies, the site of cement failure was shown to occur primarily at the band/cement interface with GIC, ${ }^{[14,15]}$ whereas failure was more common at the enamel/cement interface for RMGIC ${ }^{[7,12,14]}$ and compomers. ${ }^{[14]}$ The differences in the results of the present investigation are due to differences in methodologies. For example, we used human third molars instead of porcelain 
or gold crowns, a customized band removal device, and a different debanding location evaluation scale, crosshead speed, and specimen storage system.

Although laboratory studies to determine the retentive strength of cemented bands are important, adding a survival-time test along with SPBS testing provides more reliable information on how cemented bands hold up to externally applied mechanical stress. ${ }^{[7,14]}$ The analyses of the ball mill fatigue test have shown that it provides reproducible results in a short period of time that are consistent with the clinical performance of cements. ${ }^{[16]}$ In this study, the survival times of transbond plus and Nova Resin cements were greater than those of Ketac-Cem or GC Equia. Although R \& D series Nova Glass-LC showed a significantly higher survival time than GC Equia, there was no significant difference in survival time between R \& D series Nova Glass-LC and Ketac-Cem. Resin cement and compomer cement had greater survival times than GIC, which is consistent with some similar studies where GIC was found to have the shortest survival time. ${ }^{[7,16]}$ However, Millett et al. compared compomer (transbond plus) with GIC (Ketac-Cem) for orthodontic band cementation in and in vitro test, and they found no statistically significant difference in survival times among the adhesive cements. ${ }^{[14]}$

Results of this study suggest that although traditional GIC cement presented higher retentive strength than resin-based cements (resin, RMGIC, and compomer cement), resin based cements, especially dual cure resin cement (nova resin cement) and compomer (transbond plus), can be expected to have lower failure rates for band cementation than GIC (Ketac-Cem) in the light of the results of the ball mill test. In addition, although there was no significant difference between resin cement and compomer cement, compomer cement might be a more favorable adhesive material than resin cement for SMs with band cementation because its fluoride release capacity provides a caries protective effect. On the other hand, further studies would provide additional data that pediatric dentists could use for cement selection. Moreover, long-term clinical investigations would be ideal for comparing cements. One limitation regarding in vitro research such as the present study is duration of the study. Because bands are used clinically for months to years, it would be useful to follow cement retention characteristics beyond the initial $24 \mathrm{~h}$. A second limitation in this study was that the SM bands were cemented on dry teeth, a dry condition which, especially on the mandibular side of the children, is often difficult to achieve clinically.

\section{CONCLUSIONS}

Ketac-Cem (GIC) and R \& D series Nova Resin (dual cure resin cement) required significantly higher forces to deband in comparison with transbond plus (compomer cement) and GC Equia (GIC). Although the mean retentive strength of $R$ \& $D$ series Nova Glass-LC (RMGIC) was higher than that of transbond plus and GC Equia and lower than that of Ketac-Cem and R \& D series Nova Resin, the differences did not reach statistically significant levels. On the other hand, mean fatigue survival times of bands cemented on teeth with both transbond plus and Nova Resin cements were nearly equal and significantly longer than for bands cemented with Ketac-Cem or GC Equia. Although R \& D series Nova Glass-LC showed significantly higher survival time compared with GC Equia, there was no significant difference in survival time between $\mathrm{R} \& \mathrm{D}$ series Nova Glass-LC and Ketac-Cem.

\section{REFERENCES}

1. Chaudhary V, Shrivastava B, Bhatia HP, Aggarwal A, Singh AK, Gupta N. Multifunctional Ribbond - A versatile tool. J Clin Pediatr Dent 2012;36:325-8.

2. Laing E, Ashley P, Naini FB, Gill DS. Space maintenance. Int J Paediatr Dent 2009;19:155-62.

3. Baroni C, Franchini A, Rimondini L. Survival of different types of space maintainers. Pediatr Dent 1994;16:360-1.

4. Tannure PN, Valinoti AC, Maia LC. The use of a natural tooth crown following traumatic injuries in primary dentition. J Clin Pediatr Dent 2009;33:275-8.

5. Qudeimat MA, Fayle SA. The longevity of space maintainers: A retrospective study. Pediatr Dent 1998;20:267-72.

6. Mandall NA, Millett DT, Mattick CR, Hickman J, Macfarlane TV, Worthington HV. Adhesives for fixed orthodontic brackets. Cochrane Database Syst Rev 2003;2:CD002282.

7. Millett DT, Duff S, Morrison L, Cummings A, Gilmour WH. In vitro comparison of orthodontic band cements. Am J Orthod Dentofacial Orthop 2003;123:15-20.

8. Artun J, Bergland S. Clinical trials with crystal growth conditioning as an alternative to acid-etch enamel pretreatment. Am J Orthod 1984;85:333-40.

9. Rajab LD. Clinical performance and survival of space maintainers: Evaluation over a period of 5 years. ASDC J Dent Child 2002;69:156-60, 124.

10. Fathian M, Kennedy DB, Nouri MR. Laboratory-made space maintainers: A 7-year retrospective study from private pediatric dental practice. Pediatr Dent 2007;29:500-6.

11. Sasa IS, Hasan AA, Qudeimat MA. Longevity of band and loop space maintainers using glass ionomer cement: A prospective study. Eur Arch Paediatr Dent 2009;10:6-10.

12. Aggarwal M, Foley TF, Rix D. A comparison of shear-peel band strengths of 5 orthodontic cements. Angle Orthod 2000;70:308-16.

13. Gillgrass TJ, Benington PC, Millett DT, Newell J, Gilmour WH. Modified composite or conventional glass ionomer for band cementation? A comparative clinical trial. Am J Orthod Dentofacial Orthop 2001;120:49-53.

14. Millett DT, Cummings A, Letters S, Roger E, Love J. Resin-modified 
glass ionomer, modified composite or conventional glass ionomer for band cementation? - An in vitro evaluation. Eur J Orthod 2003;25:609-14.

15. Millett DT, Kamahli K, McColl J. Comparative laboratory investigation of dual-cured vs. conventional glass ionomer cements for band cementation. Angle Orthod 1998;68:345-50.

16. Herion T, Ferracane JL, Covell DA Jr. Three cements used for orthodontic banding of porcelain molars. Angle Orthod 2007;77:94-9.

\begin{tabular}{|l|l|}
\hline \multicolumn{2}{|c|}{ Access this article online } \\
\hline Quick Response Code: & Website: \\
\hline & www.eurjent.com \\
\hline & \\
\hline
\end{tabular}

